\title{
Treatment of Mullerian duct cyst by combination of transurethral resection and seminal vesiculoscopy: An initial experience
}

\author{
CHENKUI MIAO*, SHOUYONG LIU*, KAI ZHAO*, JUNDONG ZHU, YE TIAN, \\ YUHAO WANG, BIANJIANG LIU and ZENGJUN WANG \\ State Key Laboratory of Reproductive Medicine and Department of Urology, \\ The First Affiliated Hospital of Nanjing Medical University, Nanjing, Jiangsu 210029, P.R. China
}

Received January 6, 2018; Accepted November 7, 2018

DOI: $10.3892 /$ etm.2019.7199

\begin{abstract}
The major purpose of the present study was to investigate the efficacy and feasibility of the Mullerian duct cyst treatment by transurethral electrotomy combined with seminal vesiculoscopy. The clinical data of 20 aspermia patients who presented with Mullerian Cyst between March 2009 and March 2016 were retrospectively analyzed in the present study. Semen specimens of all patients were obtained by masturbation or sperm collector and diagnosed as aspermia by semen analysis (including sperm count, semen volume, sperm density, $\mathrm{pH}$ and fructose level). By transrectal ultrasonography, magnetic resonance imaging and testicular biopsy, the diagnosis of Mullerian cyst inducing obstruction aspermia was correctly identified. All patients were treated with the combination of transurethral resection and seminal vesiculoscopy. The operation time was 30-50 min. The follow-up duration after the operation was 12 months. All subjects included in the present study successfully underwent the operation. The semen quality of all patients was greatly improved and sperms were detected in semen specimens. The semen routine examination results of 3 consecutive follow-up exams within 12 months were within the normal range. The ejaculate volume and semen fructose levels were significantly higher than those prior to surgery $(\mathrm{P}<0.05)$. Furthermore, at 12 months post-operatively, the seminal vesicles of 6 patients were smaller than at the pre-operative stage. In conclusion, transurethral resection combined with seminal vesiculoscopy
\end{abstract}

Correspondence to: Professor Zengjun Wang or Professor Bianjiang Liu, State Key Laboratory of Reproductive Medicine and Department of Urology, The First Affiliated Hospital of Nanjing Medical University, 300 Guangzhou Road, Nanjing, Jiangsu 210029, P.R. China

E-mail: zengjunwang@njmu.edu.cn

E-mail: bjliu@njmu.edu.cn

${ }^{*}$ Contributed equally

Key words: ejaculatory duct obstruction, Mullerian duct cyst, seminal vesiculoscopy, semen quality, transurethral resection may be an effective and feasible option for the treatment of patients with Mullerian duct cyst.

\section{Introduction}

The cause of ejaculation obstruction may vary, and may be divided into congenital and acquired causes. Congenital causes include absence or atresia of vas deferens, rudimentary or absent seminal vesicles, Mullerian duct cyst or Wolffian duct cysts. Acquired causes include urinogenital infection, trauma and tumor compression (1). Among these causes, Mullerian duct cyst is relatively rare in the clinic. The majority of patients affected are treated due to infertility. A Mullerian cyst is the remnant from a secondary renal tube that was not completely degenerated during the process of embryonic development. Thin layer epithelium, which secretes cystic fluid, lines the Mullerian cyst (2). At present, affected patients are mainly treated due to prostatitis symptoms, while the inflammation symptoms, hemospermia or male infertility induced by Mullerian cyst have not received sufficient attention in the clinic.

It has been reported that transurethral ejaculatory incision was applied for the treatment of obstructive aspermia, which raised the awareness of clinicians regarding the compression of the ejaculatory duct by cysts as a type of obstructive aspermia; therefore, seminal duct obstruction became more detectable and correctable (3). At present, no consensus has been reached regarding the optimal treatment for Mullerian cysts with ejaculation tube obstruction. Therefore, the present study retrospectively analyzed 20 Mullerian cyst patients with obstruction-associated aspermia that were treated with transurethral resection combined with seminal vesiculoscopy (4). This is the first experience of treating this condition at The First Affiliated Hospital of Nanjing Medical University (Nanjing, China). The clinical efficacy and therapeutic benefits of this procedure were assessed within a follow-up period of 12 months.

\section{Patients and methods}

Patients and pre-operative examination. A total of 20 patients diagnosed with Mullerian duct cysts that received surgical treatment between March 2009 and March 2016 at the First 
Affiliated Hospital of Nanjing Medical University were enrolled in the present study. The number of patients enrolled was 20 . All patients had experienced infertility for $>2$ years following marriage. Patients were recruited if spouse-associated factors could be excluded and if no spermatozoa were detected in three consecutive semen routine examinations. Semen specimens of all patients were obtained by masturbation or sperm collector and were diagnosed as aspermia via semen analysis (including the assessment of sperm count, semen volume, sperm density, $\mathrm{pH}$ and fructose level). The patient age ranged from 22 to 38 years (median age, 29.5 years). None of the patients had any particular symptoms. The testicular size and genitalia were normal, and the vas deferens were palpable in all patients. The levels of sex hormones, including follicle-stimulating hormone, luteinizing hormone, testosterone, estradiol and prolactin, were within the normal ranges. Mature sperm production was confirmed in the testicles by testicular biopsy, and the presence of serious infection, pathogenic factors and abnormal test indicators was excluded. Cystoscopy with methylene blue staining and transrectal ultrasonography (TRUS) were applied to diagnose the prostatic midline cyst of eligible patients (Fig. 1A). Magnetic resonance imaging (MRI) also revealed the Mullerian duct cyst in proximity to or deviated from the central line of the verumontanum, indicating the seminal vesicle combined with ejaculatory duct expansion (Fig. 1B).

Surgical procedure. All of the 20 patients were placed in the dorsal lithotomy position under general or spinal anesthesia. A plasma resectoscope of F24 (Olympus Corporation, Tokyo, Japan) was selected for performing the operation, as it causes less damage to the urethra due to being thinner than the 26F resectoscope. Using this instrument, the operator is able to resect the cyst and stop the bleeding more accurately and minimize injury to the adjoining tissues with a smaller electric cutting ring compared to that of $26 \mathrm{~F}$. The anatomical landmark was then clearly recognized under direct vision (Fig. 2A). The situation of the verumontanum, external urethral sphincter and bladder neck were also carefully evaluated. Subsequently, the cyst wall was carefully resected by using thin-layer electric cutting in the vicinity of the verumontanum, and the distinct milky or yellowish-brown liquid outflow was observed (Fig. 2B). Transurethral seminal vesiculoscopy was performed using a 7-F or 8-F rigid ureteroscope (Olympus Corporation). The ureteroscope was first inserted into the prostatic urethra for initiatory observation of the verumontanum, the anatomical landmark of which usually lies in proximity to the seminal vesicle entrance (Fig. 2C). Under the guidance of the guidewire, the bilateral ejaculatory duct openings were identified in the prostatic utricle (Fig. 2D). Subsequently, the ureteroscope was inserted into the ejaculatory ducts and seminal vesicles were subjected to intermittent perfusion dilatation by using $0.90 \%(\mathrm{w} / \mathrm{v})$ sodium chloride (normal saline). The bilateral ejaculatory ducts and seminal vesicles were carefully observed on the endoscopic monitor. The seminal vesicles were clearly visible at this time, revealing that they contained multiple compartments with numerous folds in its wall and numerous small cavities with beams, as well as milky seminal plasma in the seminal vesicles (Fig. 3A and B). A bloody substance was observed in those patients with hematospermia (Fig. 3C). Furthermore, the clear region of inflammation was washed
Table I. Clinical characteristics of enrolled patients $(n=20)$ and data regarding the surgical procedure.

\begin{tabular}{lc}
\hline Clinical characteristic & Value \\
\hline Age (years) & $30(22-38)$ \\
Follow-up period (months) & 12 \\
Duration of surgery (min) & $35(19-55)$ \\
Bleeding $^{\mathrm{a}}(\mathrm{ml})$ & $7(5-12)$ \\
Duration of hospital stay (days) & $4(3-6)$ \\
\hline
\end{tabular}

Values are expressed as the mean (range). 'Quantity of bleeding that occurred during surgery.

with physiological saline until the bloody liquid was rinsed away, and the seminal vesicle cavity was then washed with normal saline containing antibiotics (levofloxacin, $5 \mathrm{mg} / \mathrm{ml}$; Fig. 3D). If polyps were present, they were removed using a laser (VersaPulse ${ }^{\circledR}$ PowerSuite $^{\mathrm{TM}} 80 \mathrm{~W}$; Lumenis, Inc., San Jose, CA, USA) and sent for pathological examination. The ureteroscope was then removed and an F18 air sac catheter was retained. The catheter was removed at 1-2 days after the operation. After the surgery, antibiotics (levofloxacin, $5 \mathrm{mg} / \mathrm{ml}$ ) were intravenously injected for 3 days to prevent infection. All patients were instructed to refrain from ejaculation for 3 weeks and were followed up at 3,9 and 12 months following surgery by routine semen analysis for 12 months.

Statistical analysis. Values are expressed as the mean \pm standard deviation or the mean (range) and the statistical calculations were performed using SPSS software (version 13.0; SPSS, Inc., Chicago, IL, USA). Differences between two groups were compared with Student's t-test. $\mathrm{P}<0.05$ was considered to indicate a statistically significant difference.

\section{Results}

Patient clinical characteristics. All of the 20 patients successfully underwent the operation combining transurethral resection and seminal vesiculoscopy without any complications including major bleeding, incontinence, epididymis or urethral rectum fistula. During the intra-operative period, the resected cysts and ejaculatory ducts were carefully identified. The duration of surgery was 19-55 min (mean, $35 \mathrm{~min}$ ), the amount of bleeding was $5-12 \mathrm{ml}$ (mean, $7 \mathrm{ml}$ ) and the duration of hospital stay was 3-6 days (mean, 4 days). All cases were followed up for 12 months to evaluate the efficacy of the treatment. The clinical characteristics of the patients enrolled and details regarding the surgical procedure are presented in Table I.

Sperm quality improves following surgery. After the surgery, the follow-up examination of the semen indicated that the semen volume of the patients increased constantly, and sperms were also observed in the semen specimens. In addition, the quality of the semen markedly improved following the 3 consecutive measurements determined over 12 months. A total of 8 patients reached normal standard by the end of the 

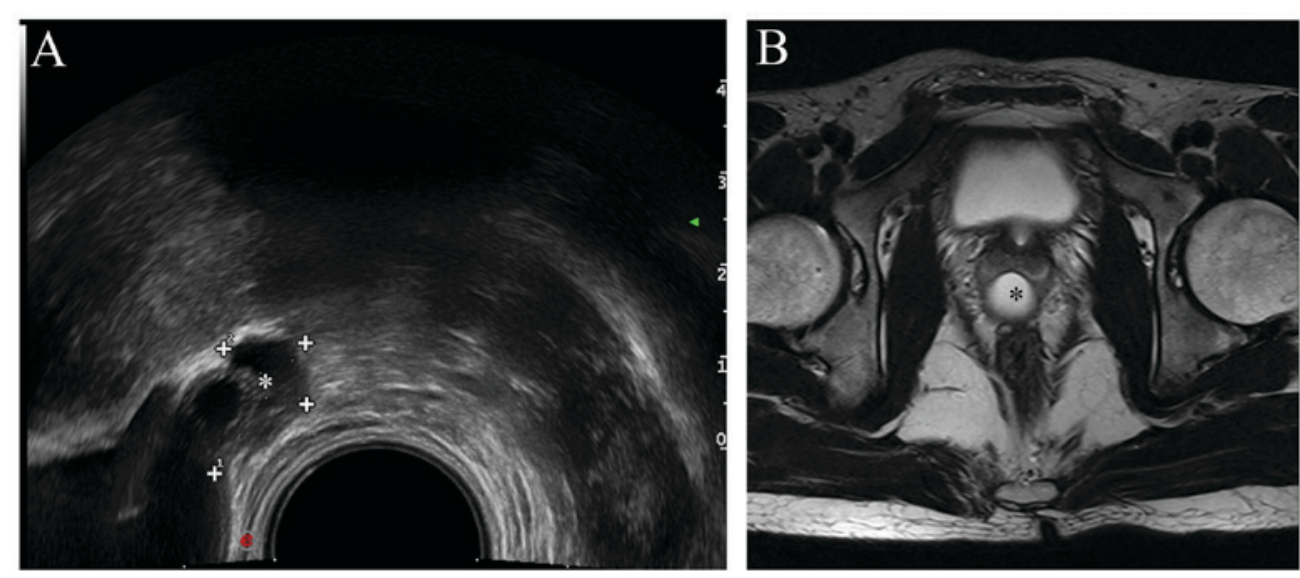

Figure 1. Imaging examination of Mullerian duct cyst. (A) Transrectal ultrasonography and (B) pelvic magnetic resonance image. The asterisk $(*)$ indicates the cystic lesion.
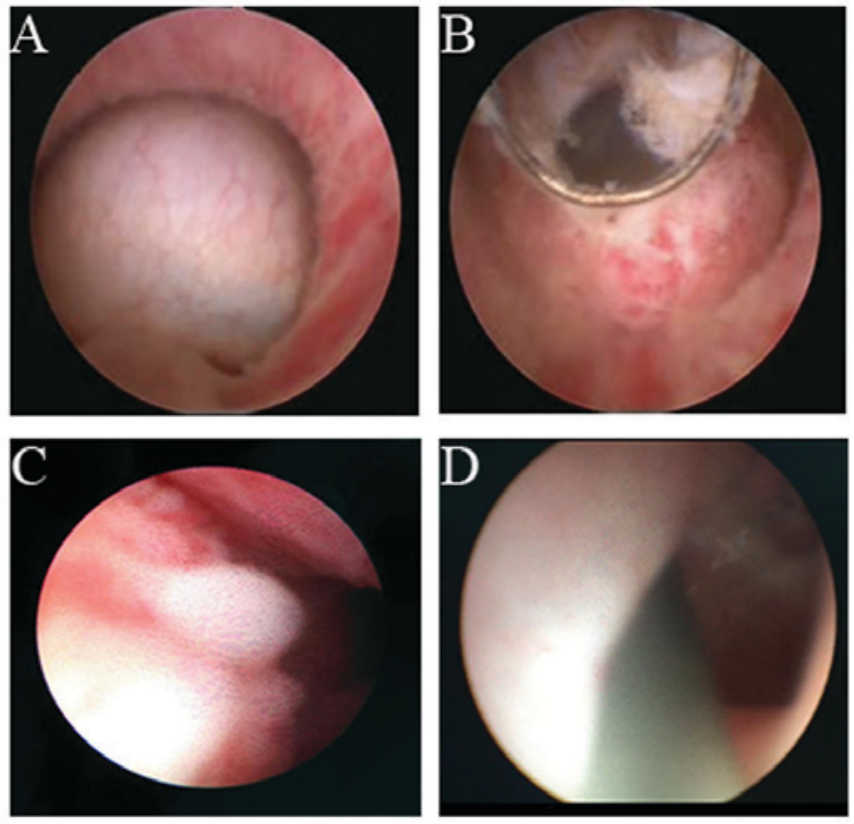

Figure 2. Resection of cyst and search of ejaculatory duct. (A) Cyst was visible under the resectoscope; (B) resection of the cyst through the resectoscope (C) observation of the verumontanum; (D) identification of the ejaculatory duct openings through the guidewire.

follow-up. Among the 20 patients, the presence of sperm was identified in the semen of 9 cases at 3 months, in 7 patients at 9 months and the remaining 4 cases at 12 months after surgery. Furthermore, the spouses of 8 patients successfully conceived at 12-15 months after surgery. In 6 patients, the volume of the seminal vesicle glands was significantly reduced after the operation. The post-operative semen volume and the seminal plasma fructose level were significantly higher than those at the pre-operative stage $(\mathrm{P}<0.05)$. A comparison of pre- and post-operative characteristics is provided in Table II.

\section{Discussion}

The clinical manifestation of a Mullerian duct cyst mainly depends on the size of the cyst, concurrent infection and secondary lesions or deformity. In clinical practice, the
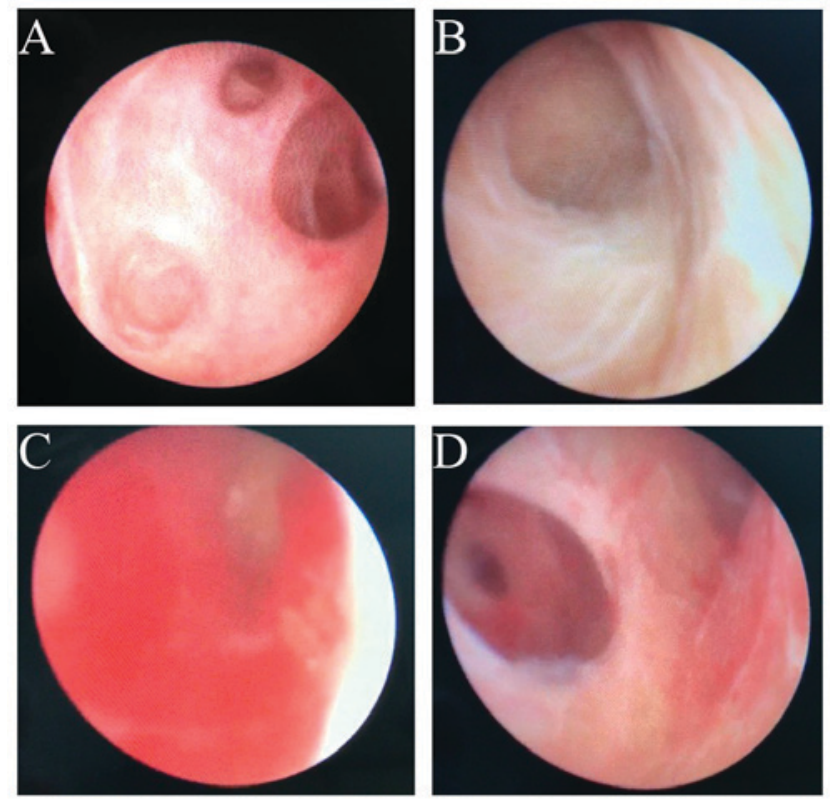

Figure 3. Observation of seminal vesicles and transurethral irrigation. (A and B) seminal vesicles contain multiple compartment with numerous folds on its wall and numerous small cavities and beams. (C and D) The seminal vesicle cavity is being cleared by flushing with normal saline containing antibiotics.

volume of most prostatic Mullerian duct cysts is low, and patients usually do not have any complaints or experience any discomfort. Mullerian duct cyst is able to cause obstruction of the ejaculation canal, thus reducing the volume of ejaculate. In addition, patients with a less severe presentation may experience discomfort in the perineum, while severe cases may present with persistent hematospermia and infertility caused by obstructive aspermia (5-7). Therefore, the enlargement of the seminal vesicle glands may be attributed to retention of excreted semen due to the blockage.

In the present study, all patients exhibited a reduced semen volume and male infertility. TRUS is able to clearly display the cystic changes in the downstream region of the prostate. Furthermore, through the location through the urethra and ejaculatory duct, it was possible to accurately diagnose the Mullerian duct cyst. The swelling of the seminal vesicle 
Table II. Comparison of the pre- and post-operative semen parameters.

\begin{tabular}{lcccc}
\hline Parameter & Pre-operation & Post-operation & P-value & Normal range $^{\mathrm{a}}$ \\
\hline Semen volume $(\mathrm{ml})$ & $1.3 \pm 0.6$ & $2.8 \pm 1.2$ & $<0.05$ & $\geq 1.5$ \\
Sperm count & 0 & $41.5 \pm 1.54$ & $<0.05$ & $\geq 39 \times 10^{6}$ \\
Sperm density $(\mathrm{g} / \mathrm{ml})$ & 0 & $15.2 \pm 2.90$ & $<0.05$ & $\geq 15 \times 10^{6}$ \\
Semen $\alpha$-glucosidase $(\mathrm{U} / \mathrm{l})$ & $13.7 \pm 5.3$ & $30.4 \pm 6.9$ & $<0.05$ & $\geq 20$ \\
Seminal plasma fructose $(\mathrm{mmol} / \mathrm{l})$ & $11.5 \pm 3.2$ & $16.2 \pm 5.4$ & $<0.05$ & $\geq 13$
\end{tabular}

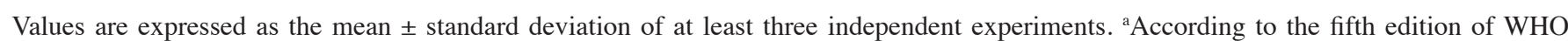
laboratory manual for the Examination and processing of human semen.

caused by ejaculatory duct occlusion was simultaneously visualized. MRI scan may also help to diagnose Mullerian duct cyst and indicate the expansion of the ejaculatory duct and seminal vesicles (8-10). The treatment of Mullerian duct cysts is mainly determined by clinical symptoms and complications associated with it. Current therapeutic options include TRUS-guided puncture and application of sclerosing agents (11-13). The surgical treatments consist of transurethral cyst incision drainage, open cyst resection and laparoscopic cystectomy (14-16). Coppens et al (17) suggested that the treatment of Mullerian duct cysts is most suitable for symptomatic or infertile patients. A smaller cyst may be observed regularly without any additional treatment. Furthermore, for large cysts that are limited to the prostate or beyond the bladder, transurethral cyst incision is a viable surgical option. However, this method may damage the verumontanum and cause urethral stricture, resulting in acute or chronic inflammation of the vas deferens, seminal vesicle and epididymis, or even occlusion. Complete obstruction may occur after transurethral resection of ejaculatory duct in patients with oligospermia or asthenospermia.

The application of transurethral resection combined with dilation of the ejaculatory duct for the treatment of Mullerian duct cyst has been rarely described. In the present study, the combination of transurethral resection and seminal vesiculoscopy was utilized to effectively avoid the possibility of probable adverse events during surgery, including not only the obstruction of the vas deferens but also relevant complications. During the surgical procedure, the relative association between cyst and prostate requires careful evaluation. The range of resection should not exceed the seminal colliculus and attention should be paid to the depth of electric resection, as well as the landmarks of the external urethral sphincter and bladder neck. As a minimally invasive procedure performed in the urogenital tract, its application may minimize the damage to the urethra to a certain extent. However, cautious and elaborate operation is still required due to the limited space in the urethra and prostate.

In conclusion, the present study provides a rational surgical approach of transurethral resection combined with seminal vesiculoscopy for the treatment of Mullerian duct cyst. Compared with the pre-operative state, patients receiving surgery treatment obtained an improvement of semen quality. The surgical approach provided in the present study is highly efficacious in treating Mullerian duct cyst with male infertility and worth recommending in clinical practice.

\section{Acknowledgements}

Not applicable.

\section{Funding}

The present study was supported by the National Natural Science Foundation of China (grant nos. 81270685 and 81771640), the Six Talent Peak Project of High-Level Talents in Jiangsu Province (grant no. WSW-017), the funded project of 333 High-level Talent Cultivation Project in Jiangsu Province, Qing Lan Project Funding of Jiangsu Higher Education Institutions (grant no. JX2161015100), A Project Funded by the Priority Academic Program Development of Jiangsu Higher Education Institutions (grant no. JX10231802), The Fifth Batch of Outstanding Young and Middle-aged Teachers Support Plan of Nanjing Medical University, Jiangsu Province's Key Provincial Talents Program (grant no. ZD RCA2016012) and the Project of the Nanjing Science and Technology Committee (grant no. 201605001).

\section{Availability of data and materials}

All data generated and/or analyzed during the present study are included in this published article.

\section{Authors' contributions}

ZW and BL conceived and designed the current study. CM, SL and KZ analyzed the data and wrote the manuscript. JZ, YT and YW collected the patient data. All authors read and approved the final version of the manuscript.

\section{Ethics approval and consent to participate}

Approval for this study was granted by the Ethics Committee of Nanjing Medical University (Nanjing, China) and written informed consent was obtained from all enrolled participants.

\section{Patient consent for publication}

Not applicable. 


\section{Competing interests}

The authors declare that they have no competing personal or financial interests.

\section{References}

1. Fisch H, Lambert SM and Goluboff ET: Management of ejaculatory duct obstruction: Etiology, diagnosis, and treatment. World J Urol 24: 604-610, 2006.

2. Kondi-Pafiti A, Grapsa D, Papakonstantinou K, Kairi-Vassilatou E and Xasiakos D: Vaginal cysts: A common pathologic entity revisited. Clin Exp Obstet Gynecol 35: 41-44, 2008.

3. Jiang HT, Yuan Q, Liu Y, Liu ZQ, Zhou ZY, Xiao KF and Yang JG: Multiple advanced surgical techniques to treat acquired seminal duct obstruction. Asian J Androl 16: 912-916, 2014.

4. Liu B,Li J,Li P,Zhang J, Song N, Wang Z and Yin C: Transurethral seminal vesiculoscopy in the diagnosis and treatment of intractable seminal vesiculitis. J Int Med Res 42: 236-242, 2014.

5. Zhao H, Luo J, Wang D, Lu J, Zhong W, Wei J and Chen W: The value of transrectal ultrasound in the diagnosis of hematospermia in a large cohort of patients. J Androl 33: 897-903, 2012.

6. Paick JS: Transurethral resection of the ejaculatory duct. Int J Urol 7 (Suppl): S42-S47, 2000.

7. Moukaddam HA, Haddad MC, El-Sayyed K and Wazzan W: Diagnosis and treatment of midline prostatic cysts. Clin Imaging 27: 44-46, 2003.

8. Pastore AL, Palleschi G, Fuschi A, Porta N, Cerbelli B, Di Cristofano C, Petrozza V and Carbone A: Hematospermia and xanthogranulomatous prostatitis: An unusual onset of a rare diagnosis. Can Urol Assoc J 7: E820-E822, 2013.

9. Furuya R, Furuya S, Kato H, Saitoh N, Takahash S and Tsukamoto T: New classification of midline cysts of the prostate in adults via a transrectal ultrasonography-guided opacification and dye-injection study. BJU Int 102: 475-478, 2008.
10. Guo Y, Liu G, Yang D, Sun X, Wang H, Deng C, Zhang Y and Feng ST: Role of MRI in assessment of ejaculatory duct obstruction. J Xray Sci Technol 21: 141-146, 2013.

11. Fisch H, Kang YM, Johnson CW and Goluboff ET: Ejaculatory duct obstruction. Curr Opin Urol 12: 509-515, 2002.

12. Cheng G, Liu B, Song Z, Xu A, Song N and Wang Z: A novel surgical management for male infertility secondary to midline prostatic cyst. BMC Urol 15: 18, 2015.

13. Han CH, Liang Q, Dong BZ, Hao L, Fan T, Zhang JJ, Zhang WD, Chen B, Qiu XZ, Zhou XJ and Pei CS: The transurethral seminal vesiculoscopy in the diagnosis and treatment of the seminal vesicle disease. Cell Biochem Biophys 66: 851-853, 2013.

14. Manohar T, Ganpule A and Desai M: Transrectal ultrasound-and fluoroscopic-assisted transurethral incision of ejaculatory ducts: A problem-solving approach to nonmalignant hematospermia due to ejaculatory duct obstruction. J Endourol 22: 1531-1535, 2008.

15. Wang H, Ye H, Xu C, Liu Z, Gao X, Hou J, Wang L, Piao S and Sun Y: Transurethral seminal vesiculoscopy using a $6 \mathrm{~F}$ vesiculoscope for ejaculatory duct obstruction: Initial experience. J Androl 33: 637-643, 2012.

16. Zhang DX, Li XG, Gao Y, Liu YS, Wang JK, Chen J, Chen L, Wang K, Cui XG and Xu DF: Transperitoneal laparoscopic excision of seminal vesicle cyst: A single-center experience. J Endourol 26: 1153-1158, 2012.

17. Coppens L, Bonnet P, Andrianne R and de Leval J: Adult müllerian duct or utricle cyst: Clinical significance and therapeutic management of 65 cases. J Urol 167: 1740-1744, 2002.

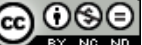

This work is licensed under a Creative Commons Attribution-NonCommercial-NoDerivatives 4.0 International (CC BY-NC-ND 4.0) License. 